\title{
Sarcoidosis with Rare Extrapulmonary Involvement
}

\author{
Marta Brandão Calçada, Sara Montezinho, Andreia M Teixeira, Bruno Gomes, Bernardo Macedo \\ Internal Medicine Department, Centro Hospitalar de Entre o Douro e Vouga, Santa Maria da Feira, Portugal
}

Received: 02/04/2021

Accepted: $13 / 04 / 2021$

Published: $12 / 07 / 2021$

How to cite this article: Brandão Calçada M, Montezinho S, Teixeira A, Gomes B, Macedo B. Sarcoidosis with rare extrapulmonary involvement. EJCRIM 2021;8: doi:10.12890/2021_002540.

Conflicts of Interests: The authors declare there are no competing interests.

This article is licensed under a Commons Attribution Non-Commercial 4.0 License

\section{ABSTRACT}

Sarcoidosis is a systemic granulomatous disease in which medullary involvement is a rare extrapulmonary manifestation. We present the case of a 37-year-old man with right abdominal and dorso-lumbar pain lasting for months. Computerized tomography showed renal microlithiasis and retroperitoneal, hilar and mediastinal adenopathies. Laboratory results showed an elevated erythrocyte sedimentation rate, IgG, $\beta 2-$ microgobulin and angiotensin-conversion enzyme, serum calcium in the upper limit and hypercalciuria. There was a slight elevation of the CD4/CD8 ratio in bronchoalveolar lavage, without lymphocytic alveolitis. An endobronchial ganglion biopsy was inconclusive. A positron emission tomography scan demonstrated supra and infra-diaphragmatic, splenic and medullary involvement, suggesting lymphoproliferative disease (LPD). A bone marrow biopsy (BMB) revealed sarcoid-like epithelioid cell granulomas, excluding LPD. Sarcoidosis was assumed and corticosteroids were started. Although cytopenias were not present, the extensive ganglion, splenic and medullary involvement made LPD exclusion imperative, while BMB allowed for a definitive diagnosis.

\section{LEARNING POINTS}

- Sarcoidosis is a multisystemic granulomatous disease in which medullary involvement is a rare extrapulmonary manifestation.

- Bone marrow biopsy is rarely performed based on serial blood counts, which may be associated with underdiagnosis.

- FDG-PET cannot reliably distinguish between sarcoidosis and lymphoproliferative disease as the cause of extensive ganglion, splenic and bone marrow involvement, and so a high index of clinical suspicion is required.

\section{KEYWORDS}

Bone marrow biopsy, bone marrow sarcoidosis, extrapulmonary sarcoidosis, sarcoidosis

\section{INTRODUCTION}

Sarcoidosis is a multisystemic granulomatous disease of unclear aetiology, characterized by tissue accumulation of CD4+ T lymphocytes and macrophages. Factors such as genetic predisposition, namely polymorphisms at the level of the human leukocyte antigen (HLA) system, and infectious or chemical environmental triggers, modulate both the incidence and clinical phenotype ${ }^{[1]}$. With an estimated prevalence of $15.3-$ 21.7 per 100,000 inhabitants, depending on different series and geographic location, it mainly affects young adults and is characterized by the histological presence of non-caseating granulomas in involved organ ${ }^{\mathrm{s}}[2,3]$.

Clinical presentation is heterogeneous, but in more than $90 \%$ of cases, it manifests with bilateral hilar adenopathies as well as signs and symptoms of pulmonary, cutaneous or ocular involvement, alone or simultaneously. Extrapulmonary involvement occurs in up to $30 \%$ of patients, but bone marrow (BM) is rarely affected ${ }^{[4,5]}$. 


\section{CASE DESCRIPTION}

A 37-year-old male patient was evaluated in an internal medicine consultation after multiple presentations to the emergency department due to right dorso-lumbar pain with months of evolution. He had no relevant past medical or surgical history, no usual medication or substance abuse habits, and no family history of rheumatological or hereditary disease. Physical examination was normal.

An abdominal ultrasound performed in an outpatient clinic documented right renal microlithiasis. This was confirmed by computerized tomography, which also showed retroperitoneal and bilateral mediastinal hilar adenopathies (the largest in the laterotracheal position, with a $13 \mathrm{~mm}$ short axis) along with subpleural and pericysural micronodules.

Laboratory analysis showed an increase in the erythrocyte sedimentation rate $(56 \mathrm{~mm} / \mathrm{hr})$, and a proteinogram showed polyclonal gammopathy and elevation of total proteins ( $8.4 \mathrm{mg} / \mathrm{dl})$, IgG (2218 mg/dl), $\beta 2$-microglobulin (3.73 mg/ml) and angiotensin-converting enzyme $(91.2 \mathrm{U} / \mathrm{I})$. Calcium was in the upper limit $(10.1 \mathrm{mg} / \mathrm{dl})$ and there was hypercalciuria (351 mg/day). Serial blood counts did not reveal any cytopenias or leukocyte count deviation. The peripheral blood smear and immunophenotyping were normal. The liver profile, renal function and urinary sedimentation rate were also normal. Viral serologies, autoimmunity study and IGRA were negative. Biopsy of minor salivary glands and ophthalmology evaluation did not show any changes.

Bronchoalveolar lavage (BAL) showed a slight CD4/CD8 ratio elevation (2.27), without lymphocytic alveolitis (11\%). Endobronchial ultrasound (EBUS) findings were non-specific and lymph node aspiration biopsy was inconclusive.

Positron emission tomography with 18F-fluorodeoxyglucose (FDG-PET) was performed for diagnostic guidance, demonstrating ganglion (supra and infra-diaphragmatic), splenic and medullary (proximal portion of the left humeral and right ischial diaphysis) hypermetabolic involvement, suggesting high metabolic grade lymphoproliferative disease (LPD) as the most likely hypothesis (Fig. 1).

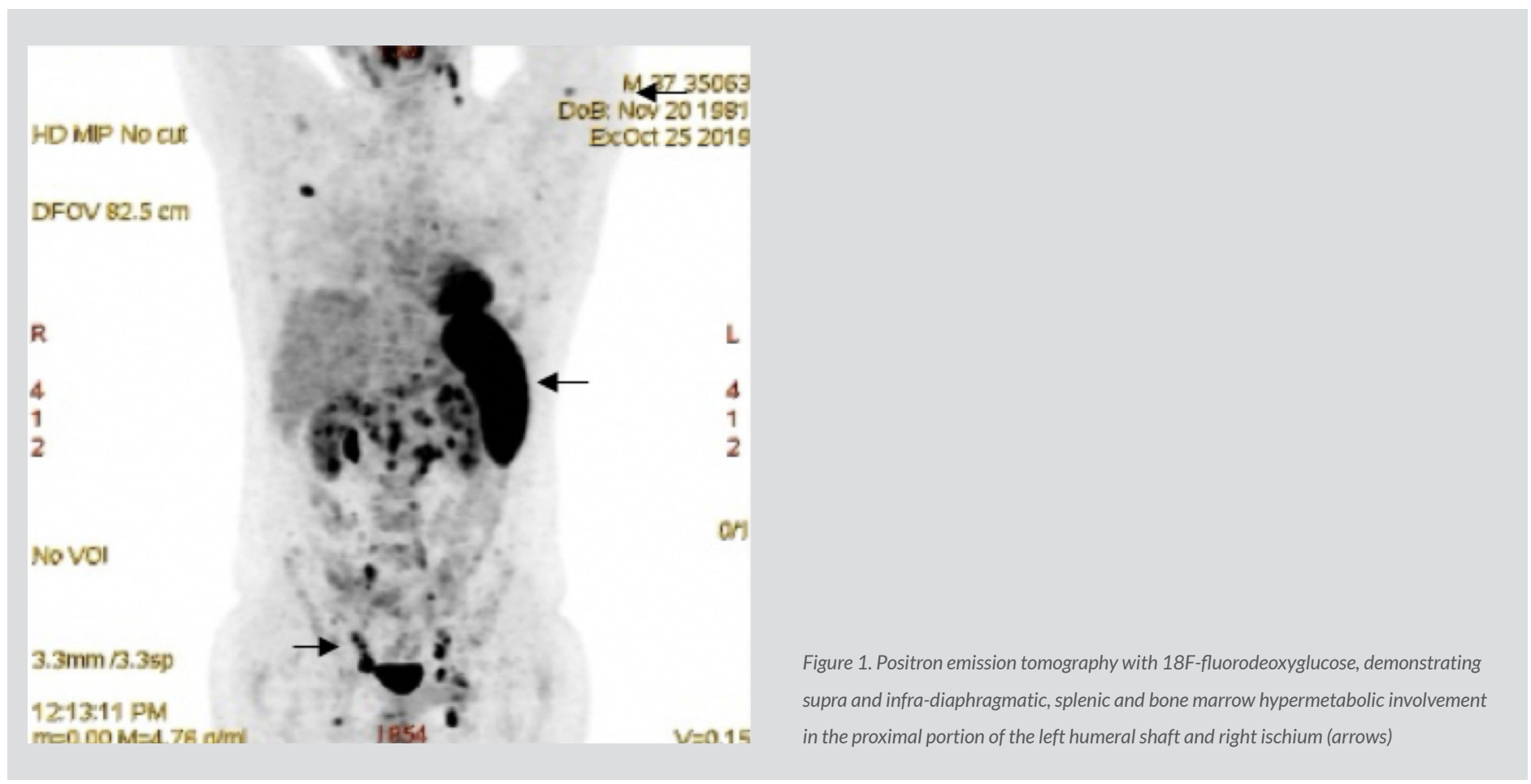

Bone marrow biopsy (BMB) excluded LPD (normocellular aspirate, with small lymphoid populations and without immunophenotypic changes suggestive of LPD) and demonstrated several granulomas composed of sarcoid-like epithelioid cells (Fig. 2).

The diagnosis of sarcoidosis with spinal and ganglionic involvement was assumed. Steroid therapy was started (prednisolone $0.5 \mathrm{mg} / \mathrm{kg} /$ day), given renal lithiasis probably secondary to calcium deposition.

Six months after the start of treatment, the patient remained asymptomatic. Steroids are currently being tapered.

\section{DISCUSSION}

Our report describes a rare extrapulmonary sarcoidosis manifestation with BM involvement. It is present in approximately $6 \%$ of cases of pulmonary sarcoidosis, but in less than $5 \%$ of cases with isolated extrapulmonary involvement ${ }^{[4,5]}$. 


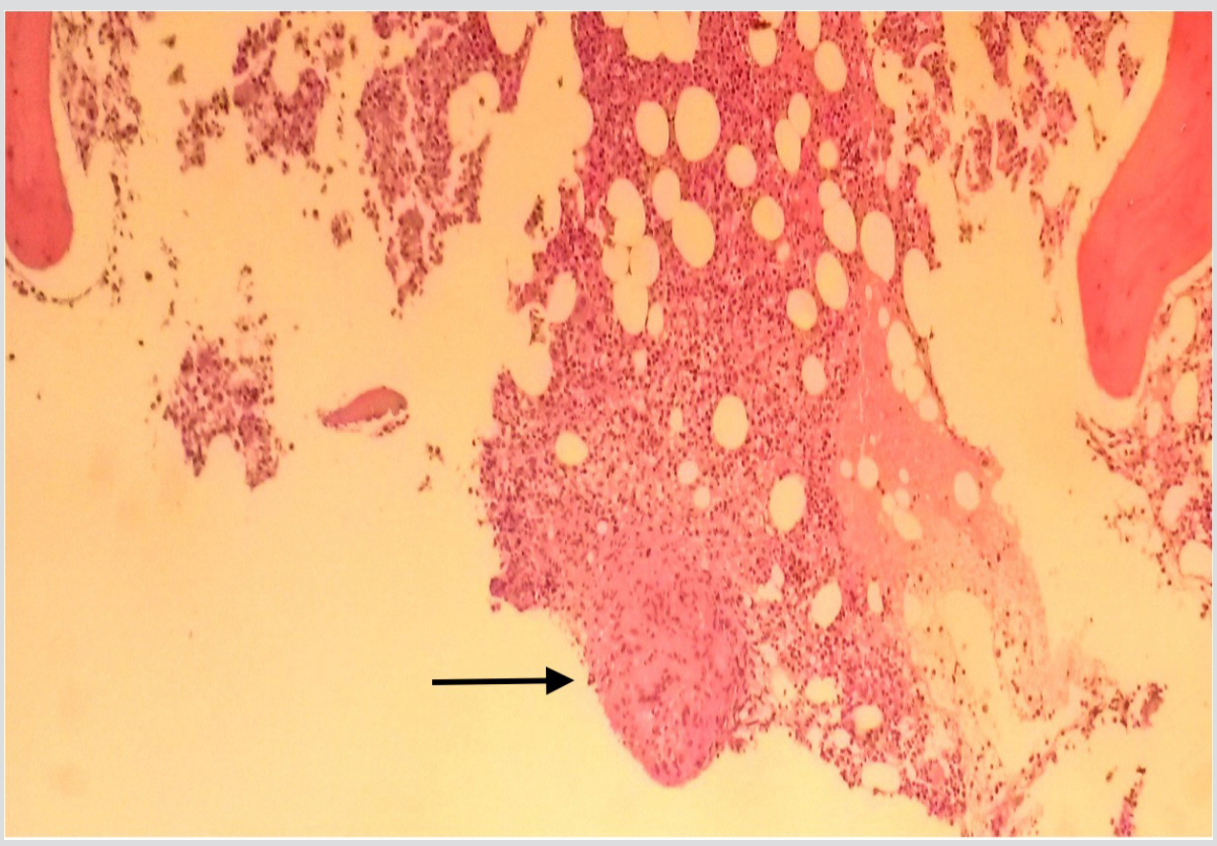

Figure 2. Non-caseous epithelioid granuloma on bone marrow biopsy (arrow) Figure 2 Non-caseous epithelioid granuloma on bone marrow biopsy (arrow)

According to the American Thoracic Society and the British Thoracic Society, despite the absence of objective measures to establish it, the diagnosis of sarcoidosis must be based on three major criteria: (i) a compatible clinical presentation; (ii) the presence of non-caseous granulomatous inflammation in one or more organ tissues; and (iii) the exclusion of alternative diagnoses of granulomatous disease ${ }^{[6,7]}$.

Regarding extrapulmonary involvement, the measurement of serum and urinary calcium is recommended, the latter in all patients with evidence of renal lithiasis, which was the first clinical manifestation in our case. Hypercalcaemia and/or hypercalciuria are due to an increase in serum levels of 1.25-(OH)2-cholecalciferol (calcitriol), following overexpression of 1-alpha-hydroxylase in granulomas ${ }^{[7]}$.

In the presence of compatible imaging findings (mediastinal and bilateral hilar adenopathies) for pulmonary sarcoidosis, as in our case, EBUS is the exam of choice with a sensitivity that varies between $54 \%$ and $93 \%{ }^{[6,7]}$.

The triad of a CD4/CD8 ratio greater than 4:1, lymphocytes greater than $16 \%$ on BAL and non-caseating granulomas in EBUS-guided ganglion biopsy, has a positive predictive value of $100 \%$ and of $81 \%$ in distinguishing sarcoidosis from pulmonary interstitial disease and other diseases, respectively ${ }^{[8]}$. In our case, BAL and EBUS did not allow the diagnosis.

Recently, the role of fluorine-18 fluorodeoxyglucose positron emission tomography (18F FDG-PET) has been recognized in the assessment of extrapulmonary extension/involvement and disease activity, as well as in monitoring therapeutic response in complex cases. Mostard et al. present a case series in which FDG-PET allowed the identification of bone and BM involvement in $34 \%$ of patients ${ }^{[9]}$. In our case, in the absence of cytopenias, the presence of extensive ganglion, splenic and BM involvement on PET, made the differential diagnosis with LPD necessary. Fluorine-18-alpha-methyltyrosine PET in combination with FDG-PET may be an alternative method to distinguish between malignancy and sarcoidosis, as reported in one study ${ }^{[10]}$. In the absence of such a high-yield diagnostic test, we performed BM biopsy in which the presence of several granulomas composed of sarcoid-like epithelioid cells supported the diagnosis of sarcoidosis. On the other hand, the absence of immunophenotypic changes suggestive of LPD rendered this diagnosis very unlikely.

Changes in haematological lines are infrequent, rarely leading to BM biopsy. This may be associated with underdiagnosis ${ }^{[11]}$. The incidence of granulomatous lesions in BM biopsy varies between $0.3 \%$ and $2.2 \%$, according to different series. Brackers et al. present a retrospective review of 9646 BM biopsies, reporting an incidence of granulomas of $0.6 \%$, of which $21 \%$ were related to sarcoidosis ${ }^{[4]}$. Granulomatous diseases with BM involvement which impose a differential diagnosis are: neoplasms, including lymphoma; mycobacteriosis, such as tuberculosis; other infectious aetiologies (fungi, cytomegalovirus, EBV and HIV); drugs and connective tissue diseases.

Corticosteroids are the first-line therapy for sarcoidosis with BM involvement, although randomized controlled studies are lacking. Other disease-modifying drugs, such as methotrexate, leflunomide and mycophenolate mofetil, have haematological toxicity as their main limitation. Patel et al. reported a case of complete response to adalimumab, a TNF inhibitor ${ }^{[12]}$.

Sarcoidosis is a challenging pathology due to the potential multisystemic involvement, making rare and atypical manifestations particularly difficult to interpret and associate with the disease. A high index of clinical suspicion is needed in order to recognize them. 


\section{REFERENCES}

Valeyre D, Prasse A, Nunes H, Uzunhan Y, Brillet PY, Muller-Quernheim J. Sarcoidosis. Lancet 2014;383(9923):1155-1167.

Sharma OP. Sarcoidosis around the world. Clin Chest Med 2008;29(3):357-363, vii.

lannuzzi MC, Rybicki BA, Teirstein AS. Sarcoidosis. N Engl J Med 2007;357(21):2153-2165.

4. Brackers de Hugo L, Ffrench M, Broussolle C, Seve P. Granulomatous lesions in bone marrow: clinicopathologic findings and significance in a study of 48 cases. Eur J Intern Med 2013;24(5):468-473

5. James WE, Koutroumpakis E, Saha B, Nathani A, Saavedra L, Yucel RM, et al. Clinical features of extrapulmonary sarcoidosis without lung involvement. Chest 2018;154(2):349356.

6. Crouser ED, Maier LA, Wilson KC, Bonham CA, Morgenthau AS, Patterson KC, et al. Diagnosis and detection of sarcoidosis. An official American Thoracic Society clinical practice guideline. Am J Respir Crit Care Med 2020;201(8):e26-e51.

7. Thillai M, Atkins CP, Crawshaw A, Hart SP, Ho LP, Kouranos V, et al. BTS Clinical Statement on pulmonary sarcoidosis. Thorax 2021;76(1):4-20.

8. Winterbauer RH, Lammert J, Selland M, Wu R, Corley D, Springmeyer SC. Bronchoalveolar lavage cell populations in the diagnosis of sarcoidosis. Chest 1993;104(2):352-361.

9. Mostard RL, Prompers L, Weijers RE, van Kroonenburgh MJ, Wijnen PA, Geusens PP, et al. F-18 FDG PET/CT for detecting bone and bone marrow involvement in sarcoidosis patients. Clin Nucl Med 2012;37(1):21-25.

10. Kaira K, Oriuchi N, Otani Y, Yanagitani N, Sunaga N, Hisada T, et al. Diagnostic usefulness of fluorine-18-alpha-methyltyrosine positron emission tomography in combination with 18F-fluorodeoxyglucose in sarcoidosis patients. Chest 2007;131(4):1019-1027.

11. Hameed OA, Skibinska M. Scar sarcoidosis with bone marrow involvement and associated musculoskeletal symptoms. BMJ Case Rep 2011;2011:bcr0220113863.

12. Patel SR. Systemic sarcoidosis with bone marrow involvement responding to therapy with adalimumab: a case report. J Med Case Rep 2009;3:8573. 